\title{
ASSESSMENT OF SALIVARY AND GINGIVAL CREVICULAR FLUID PERIOSTIN LEVELS IN CHRONIC PERIODONTITIS PATIENTS BEFORE AND AFTER NON-SURGICAL PERIODONTAL THERAPY
}

\author{
Amal A. Hussien* and Amira Maged**
}

\begin{abstract}
Introduction: Periostin is a matricellular protein highly expressed by the fibroblasts in the periodontal ligament. It is entangled in the maintenance of periodontal integrity which is affected by periodontal disease.

Aim: this study aimed to compare both GCF and salivary periostin levels during periodontal disease to their levels after non- surgical periodontal therapy.

Subjects and methods: The present study was performed on a total of 30 subjects, 13 males and 17 females. The thirty selected participants were sampled at baseline and after receiving the non-surgical periodontal treatment.

Results: In GCF, there was a statistically significant (at $\mathrm{P} \leq 0.05$ ) increase in mean Periostin level after treatment. While in saliva, there was a statistically significant (at $\mathrm{P} \leq 0.05$ ) decrease in mean Periostin level after treatment.

Conclusion Our results suggested that GCF periostin level may be considered as a reliable marker in the evaluation of periodontal disease activity, while salivary periostin level could have a promising diagnostic potential. So, further investigations are recommended.
\end{abstract}

KEYWORDS: Periostin, non-surgical periodontal treatment, saliva, Gingival crevicular fluid

\section{INTRODUCTION}

Periodontal diseases are group of inflammatory diseases caused by specific oral micro-organisms resulting in progressive destruction of the supporting tissues of the teeth ${ }^{(1)}$. It affects up to $90 \%$ of adult population worldwide (2) and is considered the primary cause of tooth loss in adults ${ }^{(3)}$. Chronic periodontitis (CP) pathogenesis is attributed to the imbalance in the host's immune inflammatory response to dental biofilm bacteria ${ }^{(1)}$.

Periostin (POSTN) is an extracellular matrix protein $(90 \mathrm{kD})$ of 835 amino acids (in humans),

*Lecturer, Oral Medicine \& Periodontology Department, Faculty of Dentistry, Cairo University, Egypt.

**Associate Professor, Oral Medicine \& Periodontology Department, Faculty of Dentistry, Cairo University, Egypt. 
originally named osteoblast specific factor 2 (OSF2 ) when first isolated from osteoblastic cell lineage in mice ${ }^{(4)}$. It was renamed as periostin to avoid its confusion with the transcription factor Cbfa1, and to indicate its expression in the periodontal ligament (PDL) and periosteum ${ }^{(5)}$.

Periostin is a secretory protein from the fasciclin I family. It is broadly expressed in collagen-rich tissues and fibrous connective tissues, with highest levels in the aorta, lower gastrointestinal tract, stomach, breast, uterus, placenta, thyroid tissue and periodontal ligament ${ }^{(6)}$.

Periostin serves as a necessary protein for the integrity, development, and maturity of the tissues and it is believed to play a key role in balancing and regulating homeostasis of the PDL (7). Expression of periostin is induced by the tumor growth factor (TGF)- $\beta$ and bone morphogenetic protein-2. It reinforces the cross-linking of collagen and improves the mechanical properties of the connective tissue ${ }^{(5,7,8,9,10)}$. Furthermore, it has been shown that expression of periostin increases during wound healing ${ }^{(11)}$. Periodontal therapy aims at the regeneration of the periodontal tissues, i.e. the restoration of their initial form, architecture, and function. It includes conventional methods such as scaling and root planing, periodontal surgery with or without osseous surgery, root conditioning agents, guided tissue regeneration, the use of different grafting materials, and their combination. ${ }^{(13)}$

The first phase of periodontal treatment is always represented by the initial preparation which primarily aims at the elimination or reduction of bacterial infection and the control of periodontal plaque-associated inflammation ${ }^{(14)}$.

Padial-Molina et al. ${ }^{(11)}$ and Balli et al. ${ }^{(12)}$ reported a positive correlation between periodontal disease severity and periostin level, while Norris et al., $2007^{(8)}$ found no significant difference between the level of periostin in periodontitis patients and in healthy controls.
There is still controversy regarding the correlation of periostin level in chronic periodontitis and healthy controls. Some recorded that there is an association between the periostin level and the severity of periodontal disease ${ }^{(11,12,15)}$. On the other hand, many other studies reported no significant difference in periostin level in patients during health and disease ${ }^{(8)}$. Moreover there is gap of knowledge about the effect of non-surgical periodontal therapy alone on GCF and salivary levels of periostin in chronic periodontitis patients.

Therefore, this study aimed to detect and compare salivary and GCF levels of periostin in chronic periodontitis before and after non-surgical periodontal treatment.

\section{SUBJECTS AND METHODS}

\section{Study population :}

The present study was implemented on a total of 30 subjects, 13 males and 17 females. All participants were recruited from the outpatient clinic of Oral Medicine and Periodontology Department, Faculty of Dentistry, Cairo University.

\section{Inclusion criteria:}

Participants were systemically free as evaluated by the aid of Dental modification of the Cornell medical index to standardize their systemic condition ${ }^{(16)}$. They had no history, symptoms, and/ or signs of infection and allergy. All subjects were in good health and had not received treatment with any antibiotics/anti-inflammatory drugs in the last 6 months.

\section{Exclusion criteria:}

Pregnant or breast-feeding women or children patients, those who use corticosteroids or other immune- suppressive drugs, and patients who had allergy to macrolide antibiotics and smokers, all were excluded from the study. 


\section{Ethical procedures:}

All subjects were informed about the detailed procedure and they were given written approval consent to sign. The study was performed between March 2016 to September 2016.

\section{Periodontal Examination}

All thirty chronic periodontitis (CP) subjects were clinically examined regarding the following clinical periodontal parameters: plaque index (PI), gingival index (GI), PD and CAL which were recorded for all patients at baseline and at 3 months after non-surgical periodontal treatment. PI was recognized by measuring the presence or absence of supra-gingival biofilm with a sweeping movement of the probe around all teeth surfaces ${ }^{(17)}$. Marginal gingival bleeding was recorded with $\mathrm{GI}^{(18)}$. PD was recorded from the free-gingival margin to the base of the periodontal pocket and CAL was measured from the cemento-enamel junction to the base of the periodontal pocket. Measurements were rounded to the highest whole millimeter using the Michigan 0 probe with Williams' markings.

These measurements were all recorded by a single calibrated examiner at six sites for all teeth mesio-buccal, mid-buccal, disto-buccal, mesiolingual, mid-lingual, and disto-lingual.

\section{Non-surgical periodontal therapy}

After initial examination, the thirty selected $\mathrm{CP}$ patients were given oral hygiene instructions for self-performed plaque control measures using soft tooth brush and inter-dental cleansing devices. Full mouth supra and sub-gingival scaling and root planing (SRP) were performed by the same operator. Sub-gingival debridement included use of ultrasonic devices* and periodontal Gracey curettes ${ }^{* *}$ under local anesthesia. The mechanical treatment included removal of all sub-gingival calcified deposits to achieve a smooth and hard surface. Each patient required one visit weekly for four successive weeks. Patients were educated to rinse with $0.12 \%$ chlorhexidine gluconate ${ }^{* * *}$ oral rinse twice daily to assist in plaque control for two weeks. Then, recall visits were made at 1 and 3 months for removal of supra-gingival plaque and reinforcing oral hygiene instructions as required. Periodontal parameters were reassessed after 3 months. GCF \& salivary samples were collected at baseline and 3 months after non-surgical periodontal therapy.

\section{Saliva collection}

Un-stimulated whole saliva was collected from all participants. Each individual was requested to abstain from eating, drinking and brushing his/her teeth for at least $60 \mathrm{~min}$ prior to collection.

Un-stimulated whole saliva was collected using the drooling technique. Each subject rinsed their mouth with water before saliva collection, and then the subject was asked to swallow to remove saliva from the mouth. The subject was seated upright, and leaned his/her head forward over a test tube with a funnel, allowing the saliva to drain into the tube. Whole saliva $(\sim 5 \mathrm{~mL})$ was obtained from each individual. During saliva collection, the test tube was placed on ice. At the end of the collection, any remaining saliva in the patient's mouth was expelled into the test tube.

Oral cavity was also evaluated to ensure the absence of debris. Using the spitting method, ${ }^{(19)}$ unstimulated saliva was collected between 11:00 am and 13:00 pm for $5 \mathrm{~min}$ (one spit per minute). The saliva was collected in sterile tubes.

\footnotetext{
* NSK non-optic ultrasonic scaler, Kanuma-shi, Japan.

** Lustra $^{\mathrm{TM}}$ Gracey periodontal curettes, Dentsply, Surrey, UK.

***Antiseptol, Kahira Pharm. Co., Egypt.
} 


\section{2-Saliva processing}

Saliva obtained was centrifuged at $15,000 \times \mathrm{g}$ (MPW-65R, MPW, Med Instrument, Warszawa, Poland) for $15 \mathrm{~min}$ at $4^{\circ} \mathrm{C}$ to remove insoluble material. Supernatant was divided into $1-\mathrm{mL}$ aliquots in pre-chilled cryo-tubes. The specimens were immediately frozen $\left(-80^{\circ} \mathrm{C}\right)$ until analysis.

\section{Collection of Gingival Crevicular Fluid (GCF)}

GCF samples were obtained from the buccal aspects of the selected site. Selection criteria for sampling sites were PD $\geq 5 \mathrm{~mm}, \mathrm{CAL} \geq 4 \mathrm{~mm}$. The selected sites were isolated by cotton rolls, rinsed gently with water and dried with a gentle air spray directed perpendicular to the gingival margin (20). A saliva ejector was used to avoid salivary contamination of the samples. Gentle removal of supra-gingival plaque was completed utilizing dry gauze, and a sterile filter paper strip (Periopaper $®$, Amityville, NY, USA) was gently inserted into the entrance of the selected site until the first sign of resistance was felt for $30 \mathrm{sec}$. Samples contaminated with gingival bleeding were excluded. The strips were placed in a dry Eppendorf tube and stored at $-80{ }^{\circ} \mathrm{C}$ until analysis.

\section{4 -Periostin level detection by ELISA:}

The level of periostin was measured in saliva and GCF samples by using ELISA kit provided by EIAab ${ }^{\circledR}$, China Catalog No: E0638h. The ELISA is based on the competitive binding Enzyme Linked Immune Sorbent Assay technique. The micro-titre plate provided in this kit has been pre coated with an antibody specific to $\mathrm{C} 4 \mathrm{a}, \mathrm{C} 4 \mathrm{a}$ in the sample or standard competes with a fixed amount of biotinlabeled $\mathrm{C} 4 \mathrm{a}$ for sites on a pre-coated Monoclonal antibody specific to C4a. Then Avidin conjugated to Horseradish was added to each well and incubated. Then a TMB substrate solution was added. Then termination was done by the addition of sulphuric acid solution and the color change was measured spectro-photo-metrically at a wavelength of $450 \mathrm{~nm} \pm 2 \mathrm{~nm}$. The concentration of $\mathrm{C} 4 \mathrm{a}$ in the samples was then determined by comparing the O.D. of the samples to the standard curve.

\section{Statistical methods:}

Data were coded and entered using the statistical package SPSS version 25. Data was summarized using mean and standard deviation for quantitative variables. Paired t-test was used to compare biochemical data and clinical periodontal parameters before and after SRP. P-values less than 0.05 were considered as statistically significant (21).

\section{RESULTS}

\section{Demographic data}

The present study was conducted on 30 patients; 13 males (43.3\%) and 17 females (56.7\%). The mean \pm standard deviation values of age were 36.3 \pm 5.1 years old with a minimum of 32.0 years and a maximum of 45.0 years old.

TABLE (1): Descriptive statistics of the different clinical parameters

\begin{tabular}{lcccccc}
\hline \multirow{2}{*}{ Parameter } & Mean baseline & SD & Mean 3 month & SD & \multicolumn{2}{c}{ 95\% CI } \\
\cline { 5 - 7 } & & & & & Lower bound & Upper bound \\
\hline GI & 2.20 & 0.79 & 0.9 & 0.2 & 1.64 & 2.76 \\
PI & 2.50 & 0.53 & 0.8 & 0.2 & 2.12 & 2.88 \\
PD $(\mathrm{mm})$ & 5.70 & 1.06 & 4.89 & 0.8 & 4.94 & 6.46 \\
CAL $(\mathrm{mm})$ & 7.00 & 1.56 & 5.00 & 0.5 & 5.88 & 8.12 \\
\hline
\end{tabular}


TABLE (2): Descriptive statistics and results of paired t-test for comparison between Periostin levels before and after treatment

\begin{tabular}{|c|c|c|c|c|c|c|}
\hline & \multirow[b]{2}{*}{ Treatment } & \multirow[b]{2}{*}{ Mean } & \multirow[b]{2}{*}{ SD } & \multicolumn{2}{|c|}{$95 \% \mathrm{CI}$} & \multirow[b]{2}{*}{$P$-value } \\
\hline & & & & Lower bound & Upper bound & \\
\hline \multirow{2}{*}{$\mathrm{GCF}$} & Pre & 85.18 & 22.35 & 69.19 & 101.17 & \multirow{2}{*}{$<0.001 *$} \\
\hline & Post & 205.53 & 74.57 & 152.19 & 258.87 & \\
\hline \multirow{2}{*}{ Saliva } & Pre & 183.74 & 16.41 & 172.00 & 195.48 & \multirow{2}{*}{$<0.001 *$} \\
\hline & Post & 133.13 & 14.12 & 123.04 & 143.23 & \\
\hline
\end{tabular}

*: Significant at $P \leq 0.05$

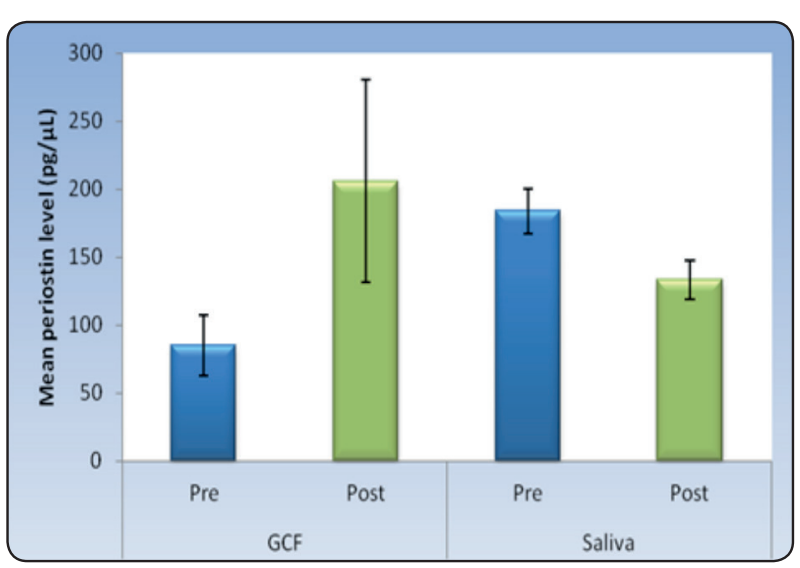

Fig. (1): Bar chart representing mean and standard deviation values of Periostin levels before and after treatment

\section{Clinical parameters}

\section{Periostin levels}

In GCF, there was a statistically significant increase in mean Periostin level after treatment. While in saliva, there was a statistically significant decrease in mean Periostin level after treatment.

\section{DISCUSSION}

GCF is an inflammatory exudate comprising wide variety of molecules. Its components have been used to detect periodontal disease activity and to distinguish patients at risk for periodontal diseases due to its simple and non-invasive collection technique ${ }^{(22)}$.

Periostin is considered as a necessary protein for tissue maturity and integrity, having a major role in periodontal ligament homeostasis ${ }^{(23)}$. Therefore, this study was conducted to correlate GCF and salivary periostin levels in patients with chronic periodontitis before and after non -surgical periodontal treatment. To the best of the author`s knowledge, this study is the first to explore such correlation.

In the current study, GCF periostin levels in patients with CP were evaluated before and after non-surgical periodontal therapy. It was found that the GCF periostin levels before treatment were lower that their levels after treatment and this finding is matching with Cüneyt et al, ${ }^{(22)}$ who compared GCF levels in $\mathrm{CP}$, aggressive periodontitis ( $\mathrm{AgP})$ to nonperiodontitis patients (NP) . Their study found that the lowest GCF periostin levels were in patients with AgP. Moreover, GCF periostin levels in CP group were lower than NP group. And similar to Balli et al, finding (12) who investigated GCF and serum levels of periostin in $\mathrm{CP}$ patients and found GCF periostin levels were reduced with the increasing in progression and severity of the disease. However, there was no significant difference in serum periostin level in their study.

Our results were also in agreement with the findings by Kumaresan et al, ${ }^{(15)}$ who compared GCF periostin levels in CP patients following non -surgical periodontal treatment with and without low level laser therapy and found that the periostin levels are significantly lower in CP patients when compared to healthy control and significantly increased in both treated groups. 
So in our study the increased levels of GCF levels after treatment confirmed a positive correlation between GCF periostin levels and the health condition of the periodontium.

Saliva is a secretion which protects the oral tissues in healthy state, has a major effect on periodontal health as it affects the plaque accumulation by its mechanical role, buffering acids originating from nutrients and bacteria, and it also has an antibacterial activity. Furthermore, saliva is a powerful tool for the characterization and advancement of periodontal disease condition and evaluating disease progression ${ }^{(24)}$.

In our study the salivary periostin levels were statistically significantly decreased after nonsurgical periodontal treatment and this finding was matched with Cüneyt et, al. ${ }^{(22)}$ who were pioneers in investigating salivary periostin levels in $\mathrm{CP}$ and $\mathrm{AgP}$ compared with healthy control and found that salivary periostin levels were highest in AgP. As compared to the $\mathrm{CP}$ and healthy control groups and explained that salivary periostin levels could be linked to gingival inflammation and periodontal disease severity in the whole mouth.

On the other hand, our results were not in agreement with the finding reported by Zeinab et al., 2018 ${ }^{(25)}$ who studied the periostin level in saliva of patient with CP compared with normal healthy and found that the salivary periostin level was significantly lower in CP than healthy controls.

This controversy reveals the need for further investigations.

\section{CONCULUSION}

Our results suggest that GCF periostin level may be considered as a trustworthy marker in assessing periodontal disease activity, while salivary periostin could have a promising diagnostic potential. Therefore, further investigations are recommended.

\section{ACKNOWLEDGEMENT}

The authors would like to acknowledge professor doctor Olfat G. Shaker professor of biochemistry, Biochemistry Department, Faculty of Medicine, Cairo University for her great efforts and skillful technical help).

\section{REFERENCES}

1. Kulkarni, C., and D.F. Kinane. (2014). Host response in aggressive periodontitis. Periodontology 2000(65): 79-91.

2. Papapanou PN (1996). Periodontal diseases: epidemiology. Ann Periodontol 1:1-36.

3. Niessen LC, Weyant RJ (1989). Causes of tooth loss in a veteran population. J Public Health Dent 49:19-23.

4. Takeshita S, Kikuno R, Tezuka K, Amann E( 1993). Osteoblast-specific factor 2: Cloning of a putative bone adhesion protein with homology with the insect protein fasciclin I. Biochem J;294:271-278.

5. Horiuchi K, Amizuka N, Takeshita S, Takamatsu H, Katsuura M, Ozawa H, et al. (1999). Identification and characterization of a novel protein, periostin, with restricted expression to periosteum and periodontal ligament and increased expression by transforming growth factor beta. J Bone Miner Res;14:1239-1249.

6. Simon J. Conway, Kenji Izuhara , Yasusei Kudo ,Judith Litvin ,Roger Markwald, Gaoliang Ouyang, Joseph R. Arron, Cecile T. J. Holweg and Akira Kudo (2014). The role of periostin in tissue remodeling across health and disease. Cell. Mol. Life Sci. 71:1279-1288

7. Padial-Molina M, Volk SL, Rodriguez JC, Marchesan JT, Galindo-Moreno P, Rios HF, et al. (2013).Tumor necrosis factor- $\alpha$ and porphyromonas gingivalis lipopolysaccharides decrease periostin in human periodontal ligament fibroblasts. J Periodontol:;84:694-703. [PubMed]

8. Norris RA, Damon B, Mironov V, Kasyanov V, Ramamurthi A, Moreno-Rodriguez R, et al. (2007). Periostin regulates collagen fibrillogenesis and the biomechanical properties of connective tissues. J Cell Biochem.;101:695-711. [PMC free article] [PubMed]

9. Ji X, Chen D, Xu C, Harris SE, Mundy GR, Yoneda T, et al. (2000). Patterns of gene expression associated with BMP-2-induced osteoblast and adipocyte differentiation of mesenchymal progenitor cell 3T3-F442A. J Bone Miner Metab.;18:132-9. [PubMed] 
10. Norris RA, Moreno-Rodriguez R, Hoffman S, Markwald $R R(2009)$. The many facets of the matricelluar protein periostin during cardiac development, remodeling, and pathophysiology. J Cell Commun Signal.;3:275-86. [PMC free article] [PubMed]

11. Padial-Molina M, Volk SL, Rios HF(2015). Preliminary insight into the periostin leverage during periodontal tissue healing. J Clin Periodontol.;42:764-72. [PubMed]

12. Balli U, Keles ZP, Avci B, Guler S, Cetinkaya BO, Keles GC, et al. (2015) Assessment of periostin levels inserum and gingival crevicular fluid of patients with periodontal disease. J Periodontal Res.; 50:707-

13. Angel Fenol, Nidhi Chinnu Boban, P. Jayachandran, Mohammed Shereef, Biju Balakrishnan, and P Lakshmi (2018). A Qualitative Analysis of Periodontal Pathogens in Chronic Periodontitis Patients afterNonsurgical Periodontal Therapy with and without Diode Laser Disinfection Using Benzoyl-DL Arginine-2-Naphthylamide Test: A Randomized Clinical Trial. Contemp Clin Dent. Jul-Sep; 9(3): 382-387.

14. Dhanangchaayan Kumaresan, Aruna Balasundaram, Vanaja Krishna Naik, Deva Priya Appukuttan(, 2017). Gingival crevicular fluid periostin levels in chronic periodontitis patients following nonsurgical periodontal treatment with low-level laser therapy. [Downloaded free from http://www.eurjdent.com on Saturday, January 07, IP: 197.124.30.123]

15. Kumaresan D, Balasundaram A, Naik VK, Appukuttan DP(2016). Gingival crevicular fluid periostin levels in chronic periodontitis patients following nonsurgical periodontal treatment with low-level laser therapy. Eur J Dent.;10:546-50. [PMC free article] [PubMed]

16. Abramson JH(1966). The cornell medical index as an epidemiological tool. Am J Public Health Nations Health. 56(2):287-98.
17. Silness J, Löe H(1964). Periodontal disease in pregnancy. ii. Correlation between oral hygiene and periodontal condtion. Acta Odontol Scand;22:121-135).

18. Löe H.(1967) The gingival index, and the retention index system.J periodontal,38:610-616

19. Navazesh M, Christensen CM(1982). A comparison of whole mouth resting and stimulated salivary measurement procedures. J Dent Res.;61:1158-62. [PubMed]

20. Griffiths, G.S.(2003).Formation, collectionand significance of gingival crevice fluid. Periodontol 200 31:32-42.

21. Chan YH (2003): Biostatistics102: Quantitative Data Parametric \& Non-parametric Tests. Singapore Med J.;44(8): 391-396.

22. Cüneyt A. Aral, Serhat Köseoğlu, Mehmet Sağlam, Tuğba Pekbağrıyanık, and Levent Savran(2016). Gingival Crevicular Fluid and Salivary Periostin Levels in NonSmoker Subjects With Chronic and Aggressive Subjects With Chronic and Aggressive Periodontitis. Inflammation, DOI: 10.1007/s10753-016-0328-0 published online :01 March 2016.

23. Padial-Molina M, Volk SL, Taut AD, Giannobile WV, Rios $\mathrm{HF}(2012)$. Periostin is down-regulated during periodontal inflammation. J Dent Res.;91:1078-84. [PMC free article] [PubMed]

24. Salazar, M.G., N. Jehmlich, A. Murr, V.M. Dhople, B. Holtfreter, E. Hammer, U. Volker, and T. Kocher (2013). Identification of periodontitis associated changes in the proteome of whole human saliva bymass spectrometric analysis. Journal of Clinical Periodontology 40:825-832.

25. Zeinab Rezaei Esfahrood, Sahar Tehrani Vardian, Zahra Yadegari, Muna Adhim, and Najmeh Sadat Valed Saravi (2018). Periostin levels in saliva of patients with chronic periodontitis. J Indian Soc Periodontol. Jan-Feb; 22(1): 25- 